\title{
Comparison of Activated Bleaching Effects of Various Laser Systems: An In vitro Study
}

\author{
Ozgul Baygin ${ }^{1 *}$, Fatih Mehmet Korkmaz ${ }^{2}$ and Ipek Arslan ${ }^{1}$
}

${ }^{1}$ Department of Pediatric Dentistry, Faculty of Dentistry, Karadeniz Technical University, Trabzon, Turkey ${ }^{2}$ Department of Prosthodontics, Faculty of Dentistry, Karadeniz Technical University, Trabzon, Turkey

\begin{abstract}
Aim: The aim of this in vitro study is to describe a preclinical investigation using laser-activated bleaching agent for discolored teeth.

Materials and methods: Sixty sound human central and lateral incisors were selected for this study and were randomly assigned into four groups as follows: Group 1 ( $n=15)$ : only Whiteness HP 10 min; Group 2 ( $n=15)$ : diode laser, wavelength $980 \mathrm{~nm}$ power $0.8 \mathrm{~W}-30 \mathrm{sec}+$ Whiteness HP; Group $3(n=15)$ : diode laser, wavelength $980 \mathrm{~nm}$ power $1 \mathrm{~W}-30 \mathrm{sec}+$ Whiteness HP; Group $4(\mathrm{n}=15)$ : KTP laser wavelength $532 \mathrm{~nm}$ power $1 \mathrm{~W}-30$ sec+Whiteness HP. In the bleaching experiment, $38 \%$ hydrogen peroxide was used. The results of the irradiations were characterized using the CIEL*a*b system.
\end{abstract}

Results: A mean total color difference value greater than 5.0 was obtained in each group. Lasers systems together with Whiteness HP bleaching gel showed the best results using only Whiteness HP gel $(p<0.05)$.

Conclusion: Application of laser systems with Whiteness HP provides simple, short, and powerful bleaching in dental offices.

Keywords: Bleaching; Whitening; Color measurement; Laser

\section{Introduction}

Bleaching of intrinsic or extrinsic teeth stains has continued to increase in popularity as an effective, noninvasive method to alter the shade of discolored teeth [1]. To accelerate the bleaching process, heat activation can be used and several safe techniques have been improved. One accepted procedure is to use a peroxide compound placed on the tooth surface that bleaches the intrinsic tooth pigments to a lighter hue. Adding heat, light, or laser energy to the peroxide compound may accelerate this process [2].

To accelerate the office bleaching procedure, several different types of irradiation sources exist representing coherent or incoherent light in use [3]. The advantages of these techniques are speed and convenient. The general handicap of dental bleaching is increased tooth or gingival sensitivity. Irradiation using visible and infrared light can provoke beneficial effects at certain wavelengths [4]. Because resin-curing lights are mostly used for bleaching, some cases have an extra setting. Light systems designed for bleaching procedures output light for an entire dental arch. A laser system mechanism offered for bleaching purposes depends on wavelength, power of radiation, and pump mode [2].

Office bleaching procedures use different types of energy sources to increase the rate of chemical release of bleaching radicals [5]. Direct heating has become less preferred over time and have been replaced by other energy sources, such as plasma-arc devices, halogen lamps, InGaN LED, or light emitting diodes, and other light sources. Lamps that emit long wavelengths as visual spectrum or larger invisible infrared spectrum have lower energy photons, causing high thermal character that may induce unfavorable thermal effects $[2,4,6-8]$. KTP, argon, and diode lasers are typically used for in-office bleaching treatments. Shorter wavelengths, such as the argon laser $(\lambda=514.5$ $\mathrm{nm}$ ) or the KTP laser (532 nm), have higher energy photons and less direct thermal characteristics. In contrast, such laser systems provide complete safety at these wavelengths for both vital and nonvital teeth when used for photobleaching $[2,4,9]$.

Dental tooth shade evaluation by humans is prone to environmental and physical variables such as ambient light, differences in the number of cones and rods within the retina of the eye, and many others $[10,11]$ Therefore, a significant aim of color vision research is the specification of human color response because electronic color measurement devices are being used. A recent important development is the CIEL $L^{*} a^{*} b$ color system [12] that allows for a difference between colors to be calculated as the Euclidean distance between their coordinates in three-dimensional $C I E L^{\star} a^{\star} b$ space. The last three letters in the name $C I E L^{\star} a^{\star} b$ refer to the three perpendicular directions in color space: $a^{*}$ represents the red-green contrast (positive a values correspond to a carmine red and negative a values correspond to its opposite, blue green); $b^{*}$ represents the yellow-blue contrast (positive $b$ values correspond to a carmine red and negative $b$ values correspond to deep blue); and $L^{*}$ corresponds to the luminosity dimension or whiteness that ranges from 0 (pure black) to 100 (reference white) and is proportional to luminance. The chroma $C^{*}$, or color saturation, which indicates the distance from the gray axis $\left(L^{*}\right)$ in $C I E L^{*} a^{*} b^{*}$ space, represents the change from unsaturated (dull) to saturated (bright) colors. A larger $C^{*}$ indicates more saturation of the color. Therefore, the two attributes that define the perceived increase in brightness succeeded by the bleaching procedure are the change in whiteness $\left(\Delta L^{*}\right)$ and the change in chroma $\left(\Delta C^{*}\right)[4]$.

Color intensity (chroma) seems like light intensity (whiteness) for humans, especially for the color yellow, which reaches its strongest saturation at high whiteness values, as represented in the Munsell color

*Corresponding author: Ozgul Baygin, Karadeniz Technical University, Faculty of Dentistry, Department of Pediatric Dentistry, Trabzon, Turkey, Tel: 00904623774780; Fax: 00904623253017; E-mail: dtozgul@gmail.com

Received July 23, 2012; Accepted August 11, 2012; Published August 13, 2012

Citation: Baygin O, Korkmaz FM, Arslan I (2012) Comparison of Activated Bleaching Effects of Various Laser Systems: An In vitro Study. Dentistry 2:139. doi:10.4172/2161-1122.1000139

Copyright: (C) 2012 Baygin O, et al. This is an open-access article distributed under the terms of the Creative Commons Attribution License, which permits unrestricted use, distribution, and reproduction in any medium, provided the original author and source are credited. 
system [4]. The Munsell system is based on a cylindrical coordinate system. However, the $C I E L^{*} a^{*} b^{*}$ system is based on a rectilinear Cartesian coordinate system that permits a description and algebraic manipulation of color differences between coordinates in color space [4]. This study uses spectrophotometric comparison to analyze the efficacy of laser systems using the HP Whiteness bleaching agent.

\section{Materials and Methods}

For the study, 60 healthy human maxillary central and lateral incisors extracted from adult males and females for various reasons were selected. The teeth were kept hydrated in saline solution. For the experiment, they were cleaned with pumice in the laboratory. The roots were separated using a diamond saw (model $15 \mathrm{HC}$, Buhler), the pulpal tissue was removed, and the dentin sealed off with self-curing epoxy. The teeth were then stored in distilled water under refrigeration at $8^{\circ} \mathrm{C}$ to keep them hydrated. All teeth had their $L^{*} a^{*} b^{*}$ values measured for the first time using a spectrophotometer (model Cintra 10, GBC Scientific Equipment, Australia) and were subsequently immersed in a staining solution made of tobacco, black tea, coffee, Coca-Cola, and red wine for seven days at a temperature of $37^{\circ} \mathrm{C}$. The receptacle containing the staining liquid was stirred once every day to avoid decantation of the staining products. After this period, the teeth were rinsed for the second time and the $C I E L^{*} a^{*} b^{*}$ values were measured again.

The teeth were divided into four groups, as follows.

Control group (Group 1): An approximately $2 \mathrm{~mm}$ thick layer of the bleaching agent (Whiteness HP) was applied and kept for 10 minutes on the control group teeth surfaces.

Group 2 received a $2 \mathrm{~mm}$ layer of the bleaching agent, which was immediately irradiated with $1 \mathrm{~W}$ of output power from the diode laser (wavelength $970 \mathrm{~nm}$ ) for 5 minutes.

Group 3 received a $2 \mathrm{~mm}$ layer of the bleaching agent, which was immediately irradiated with $2 \mathrm{~W}$ of output power from the diode laser (wavelength $970 \mathrm{~nm}$ ) for 2.5 minutes.

Group 4 received a $2 \mathrm{~mm}$ layer of the bleaching agent, which was immediately irradiated with $1 \mathrm{~W}$ of output power from the diode laser (wavelength $532 \mathrm{~nm}$ ) for 30 seconds.

The $L^{*} a^{*} b^{*}$ values were measured for the last time and then the differences between the three CIEL $\mathrm{a}^{*} \mathrm{~b}^{\star}$ measurements were evaluated. To verify whether the bleaching agent and the light source affected significantly the mean values, an analysis for multiple comparisons using Fisher's Least Significant Difference (LSD) method was applied with a $95 \%$ confidence interval $(\mathrm{P}<0.05)$.

\section{Results}

The lightness $L^{*}$ values dropped significantly from the first measurement to the measurement after the staining procedure and none of the procedures from G1 to G4 recovered to the initial values. The same occurred with the $a^{*}$ and $b^{*}$ values. All teeth showed a predominantly yellow hue, with a $b^{*}$ value between 11 and 22 , and only a slight contribution from the transverse color dimension red, with a positive $a^{\star}$ value between 1.1 and 5.1. The chroma value, which is proportional to the saturation of the color, was calculated using the Formula $C^{*}=\left(a^{*}+b^{*}\right)^{1 / 2}$. The mean values of $L^{*}$ and $C^{*}$ and their standard deviations are shown in tables 1 and 2 . For a more accurate analysis, the results were separated according to light source. The differences in $L^{*}$ and $C^{*}$ introduced by the respective treatment procedures from groups G1 to G4 were also analyzed.
The "light source" factor is also significant with respect to chroma. The mean change in chroma for both combinations using laser was significantly better than for using only a bleaching agent. Group G1 showed the smallest chroma change and, therefore, moved less in the direction of the chromatic gray axis ( $L^{*}$ axis) of the CIEL ${ }^{*} a^{*} b^{*}$ color sphere.

\section{Discussion}

The aim of laser bleaching is to achieve the perfect bleaching process with the most efficient source while avoiding any adverse effect. Argon, $\mathrm{CO}_{2}$, and $980 \mathrm{~nm}$ GaAlAs diode laser wavelengths are cleared by the FDA for tooth whitening, and other laser radiation systems have been tested. In office bleaching, KTP, Agon, and Diode laser have been preferred. KTP and the Argon laser are also completely safe at these wavelengths for both vital and nonvital tooth structures, and are feasible for photobleaching [13-15].

KTP laser (kalium-titanyl-phosphate crystal frequency doubled Nd:YAG laser, pulsed) has been absorbed relatively low in water and tooth mineral, high in hemoglobin, penetrated medium into dental hard tissues. KTP laser energy also induces a photochemical reaction in the bleaching gel similar to the photochemical reaction induced by Argon laser light in the photopolymerization of light cured materials. KTP laser energy creates acceptable temperature changes in the dental pulp and is more active in heating the surface gel. The KTP laser with high energy densities decreased the time needed to bleach teeth [13-17]. Walsh and Liu demonstrated that the whitening effect of photochemical KTP laser bleaching is more effective than diode laser photothermal bleaching. Therefore, the effect of a $532 \mathrm{~nm}$ KTP laser has a synergic effect with a diode laser. For these reasons, a $532 \mathrm{~nm} \mathrm{KTP}$ laser was used in the present study.

Zhang et al. [9] studied the whitening efficacy of Light-Emitting Diode (LED), diode laser, and KTP laser irradiation in dental bleaching by analyzing the change in color, the temperature increase in the pulp cavity, and the microhardness measurement after treatment. The author suggests that the KTP laser is effective in brightening teeth. Within the limits of this study, the LED and the KTP laser induced a safer pulpal temperature increase when assisted with Hi-Lite bleaching gel.

Diode laser absorption in water, tooth mineral, and pigments is low but in dental hard tissue is high, indicating that pulp damage from a temperature increase can occur. Most of the studies on diode lasers show that the bactericidal effect of this laser is perfect, and that it is commonly used for in-office bleaching treatments [4,18-21].

Few published studies analyzed the efficacy of laser used in a bleaching procedure. With the aim of reducing the possible reading bias in a dental office that has several types of light sources, a spectrophotometer analyzes the samples under only one light source. A spectrophotometer's reference is the light of the day, which is not always recognized by the human eye primarily if the metamerism of the object is to be considered [4]. Regarding the $L^{\star} a^{\star} b^{\star}$ values obtained in this study, high $L^{*}$ values corresponding to a higher luminosity are the lowest ones associated with less clarity, which is not expected after the dental bleaching procedure. Regarding the chroma, higher $C^{\star}$ values corresponding to a higher saturation are the lowest values (less saturation) next to a chromatic gray color $\left(C^{*}=0\right)$. Because the chroma value is linked to the luminosity value $L^{\star}$ by means of the color sphere, the higher the luminosity, the smaller the maximum possible saturation. For the highest luminosity value, there is no hue and $C^{\star}$ has to be zero (completely white teeth, top of the color sphere, $L^{*}=100$ ). For values of 


\begin{tabular}{|l|l|l|l|l|}
\hline Agent & Irradiation & Initial & Stained & Final \\
\hline \multirow{3}{*}{ Whiteness HP } & Group 1 & $90.8 \pm 1.3$ & $77.6 \pm 7.7$ & $80.6 \pm 8.1$ \\
\cline { 2 - 5 } & Group 2 & $90.9 \pm 1.7$ & $84.3 \pm 5.9$ & $89.3 \pm 2.1$ \\
\cline { 2 - 5 } & Group 3 & $91.0 \pm 1.4$ & $85.9 \pm 6.1$ & $90.2 \pm 2.0$ \\
\cline { 2 - 5 } & Group 4 & $91.1 \pm 1.3$ & $86.1 \pm 7.3$ & $91.4 \pm 1.2$ \\
\hline
\end{tabular}

Table 1: Mean Values and Standard Deviation of $L^{*}$.

\begin{tabular}{|l|l|l|l|l|}
\hline Agent & Irradiation & Initial & Stained & Final \\
\hline \multirow{3}{*}{ Whiteness HP } & Group 1 & $11.8 \pm 1.4$ & $22.6 \pm 3.7$ & $16.6 \pm 3.1$ \\
\cline { 2 - 5 } & Group 2 & $11.9 \pm 2.3$ & $25.3 \pm 4.9$ & $18.8 \pm 4.3$ \\
\cline { 2 - 5 } & Group 3 & $12.1 \pm 1.5$ & $19.9 \pm 5.4$ & $19.6 \pm 1.1$ \\
\hline & Group 4 & $12.7 \pm 1.1$ & $23.1 \pm 3.3$ & $20.4 \pm 1.8$ \\
\hline
\end{tabular}

Table 2: Mean Values and Standard Deviation of $C^{*}$

$L^{*}$ larger than 95 , the only hue that can be strongly saturated is yellow [22]. Hasegawa et al. [23] studied natural tooth color on 87 subjects in Japan and measured a mean $L^{*}$ value of 73 and a mean $b^{*}$ value of 16.5. At this $L^{*}$ value, strong saturation of almost all hues is possible and, therefore, the chroma value is an issue. According to Gerlach et al. [24] $\Delta C$ is the primary response variable because its directional measurement has the most perceptual relevance. For the $\Delta C^{*}$ values, a more accurate analysis is probable when studying human teeth for which the yellow color predominates through the transparency of the dentin in normal conditions. In addition, the greater the saturation, the brighter the teeth appear to the human eye; however, grayish-colored teeth $\left(C^{*}=0\right)$ are mostly unacceptable to patients [25]. This research produced significant differences between the results of the agent/light source associations.

To respect the lightness $L^{*}$ and the chroma $C^{*}$ values, the best results were attained with the Whiteness HP bleaching gel and diode KTP association. The Whiteness HP bleaching agent always shows a more efficient interaction with the laser.

Within the limits of the present study, laser and the Whiteness HP bleaching agent association showed more effective results with respect to both chroma and luminosity. Further studies are necessary and are being conducted to prove these results.

\section{References}

1. Sulieman M, Addy M, Rees JS (2005) Surface and intra-pulpal temperature rises during tooth bleaching: an in vitro study. Br Dent J 199: 37-40.

2. Buchalla W, Attin T (2007) External bleaching therapy with activation by heat, light or laser--a systematic review. Dent Mater 23: 586-596.

3. Luk K, Tam L, Hubert M (2004) Effect of light energy on peroxide tooth bleaching. J Am Dent Assoc 135: 194-201.

4. Wetter NU, Barroso MC, Pelino JE (2004) Dental bleaching efficacy with diode laser and LED irradiation: an in vitro study. Lasers Surg Med 35: 254-258.

5. Hein DK, Ploeger BJ, Hartup JK, Wagstaff RS, Palmer TM, et al. (2003) Inoffice vital tooth bleaching--what do lights add? Compend Contin Educ Dent 24: 340-52.

6. Eldeniz AU, Usumez A, Usumez S, Ozturk N (2005) Pulpal temperature rise during light-activated bleaching. J Biomed Mater Res B Appl Biomater 72: 254259 .

7. Walsh LJ (2003) The current status of laser applications in dentistry. Aust Dent J 48: 146-55.

8. Lizarelli RF, Bregagnolo JC, Lizarelli RZ, Palhares JM, Villa GE (2004) A comparative in vitro study to diagnose decayed dental tissue using different methods. Photomed Laser Surg 22: 205-210.

9. Zhang C, Wang X, Kinoshita J, Zhao B, Toko T, et al. (2007) Effects of KTP laser irradiation, diode laser, and LED on tooth bleaching: a comparative study. Photomed Laser Surg 25: 91-95.
10. Horn DJ, Bulan-Brady J, Hicks ML (1998) Sphere spectrophotometer versus human evaluation of tooth shade. J Endod 24: 786-790.

11. Joiner A (2004) Tooth colour: a review of the literature. J Dent 32: 3-12.

12. CIE 15.2-Calorimetry (1986) Commission Internationale De L'eclairage, Vienna, Austria.

13. Garber DA (1997) Dentist-monitored bleaching: a discussion of combination and laser bleaching. J Am Dent Assoc 128: 26S-30S.

14. Vanderstricht K, Nammour S, De Moor R (2009) [“Power bleaching” with the KTP laser]. Rev Belge Med Dent(1984) 64: 129-139.

15. Torres CR, Batista GR, Cesar PD, Barcellos DC, Pucci CR, et al. (2009) Influence of the quantity of coloring agent in bleaching gels activated with LED/ laser appliances on bleaching efficiency. Eur J Esthet Dent 4: 178-186.

16. Goharkhay K, Schoop U, Wernisch J, Hartl S, De Moor R, et al. (2009) Frequency doubled neodymium:yttrium-aluminum-garnet and diode laseractivated power bleaching--pH, environmental scanning electron microscopy, and colorimetric in vitro evaluations. Lasers Med Sci 24: 339-346.

17. Kinoshita J, Jafarzadeh H, Forghani M (2009) Vital Bleaching of TetracyclineStained Teeth by Using KTP Laser: A Case Report. Eur J Dent 3: 229-32.

18. Walsh LJ, Liu JY, Verheyen P (2004) Tooth Discoloration and Its Treatment Using KTP Laser-assisted Tooth Whitening. J Oral Laser Applications 4: 7-21.

19. Zhang C, Wang X, Kinoshita J, Zhao B, Toko T, et al. (2007) Effects of KTP laser irradiation, diode laser, and LED on tooth bleaching: a comparative study. Photomed Laser Surg 25: 91-95.

20. Dostalova T, Jelinkova H, Housova D, Sulc J, Nemec M, et al (2004) Diode laser-activated bleaching. Braz Dent J 15: SI3-S18.

21. Al Quran FA, Mansour Y, Al-Hyari S, Al Wahadni A, Mair L (2011) Efficacy and persistence of tooth bleaching using a diode laser with three different treatment regimens. Eur J Esthet Dent 6: 436-445.

22. Long J (2001) The new munsell student color set.2nd edition. Virginia: Virginia Common wealth University.

23. Hasegawa A, Motonomi A, Ikeda I, Kawaguchi S (2000) Color of natural tooth crown in Japanese people. Col Res Appl 25: 43-48.

24. Gerlach RW, Gibb RD, Sagel PA (2000) A randomized clinical trial comparing an novel $5.3 \%$ hydrogen peroxide whitening strip to $10 \%, 15 \%$, and $20 \%$ carbamide peroxide tray based bleaching systems. Compend Contin Educ Dent Suppl: S22-S28.

25. Odioso LL, Gibb RD, Gerlach RW (2000) Impact of demographic, behavioral and dental care utilization parameters on tooth color and personal satisfaction. Compend Contin Educ Dent S35-S41. 